Methods Children aged 1-16 yrs. old from primary, secondary and tertiary care in the Connacht area were invited; recruitment is ongoing; a target of 100 patients has been set. Participants are screened via a detailed telephone consultation using a non-standardised questionnaire to ensure they meet the inclusion criteria: single, non-immediate $(>1$ hour) skin rash or other symptoms compatible with an allergic reaction after beta-lactam including urticaria or angioedema occurring $>1$ hour and persisting $>12$ hours. Exclusion criteria are: immediate allergic reaction $<1$ hour; or $>1$ hour but resolving within 12 hours; cardiorespiratory involvement; multiple antibiotic allergy, atypical/severe drug reaction or IV beta-lactams and insufficiently controlled asthma. Eligible candidates attend an outpatient visit, are confirmed as clinically well, including pre-challenge temperature and cardio-respiratory exam. A single, age-appropriate dose of the causative beta-lactam is administered. Visual assessment is performed every 30 minutes for 2 hours; adverse events recorded and managed accordingly. A 5 -day beta-lactam course is prescribed to negative challenge participants. Delayed hypersensitivity and parental satisfaction is assessed via follow up phone-call 6 days post-challenge. Study approval by the hospital clinical research ethics committee was obtained.

Results To date, 76 children (16 months to 15 years) have been enrolled; 59 have undergone pre-challenge screening; with skin rash being the main indication $(53 / 59,90 \%)$. Six/seventy-six (8\%) were excluded, one withdrew; 17 children are from secondary care, 55 from primary care, 4 self-referrals. Twenty-five children have completed the challenge (24 amoxicillin, 1 flucloxacillin) with no reactions. Parents reported high satisfaction with the challenge process and confidence in using a beta-lactam antibiotic in the future.

Conclusion This study demonstrates that performing a streamlined, single dose, oral challenge to low risk patients in an outpatient setting is feasible, safe and acceptable to participants. This method may be reproducible in primary care.

\section{GP5 THE IMMUNOGENETICS AND RISK FACTORS OF POLLINOSIS AMONG RUSSIAN CHILDREN. CASE- CONTROL STUDY}

\begin{abstract}
1,2 Julia Levina*, ${ }^{3,2}$ Leyla Namazova-Baranova, ${ }^{1}$ Kirill Savostyanov, ${ }^{1}$ Alexander Pushkov, ${ }^{1}$ Alexey Burdennyy, ${ }^{1,2}$ Anna Alekseeva, ${ }^{1,3,2}$ Kamilla Efendieva, ${ }^{1,3,2}$ Elena Vishneva. ${ }^{1}$ National Medical Research Center of Children's Health, Moscow, Russian Federation; ${ }^{2}$ Central Clinical Hospital of Russian Academy of Sciences, Moscow, Russian Federation; ${ }^{3}$ Pirogov Russian National Research Medical University, Russian Academy of Sciences, Moscow, Russian Federation
\end{abstract}

\subsection{6/archdischild-2019-epa.72}

Background and aim Pollinosis is an allergic disease that manifests during spring or summer with symptoms of allergic rhinitis, conjunctivitis, asthma and atopic dermatitis. In Russia the most common reason for pollinosis is birch and grass pollen. Since pollinosis is a multifactorial disease, the aim of this study was to investigate the association between polymorphic markers of candidate genes and the development of the disease.

Methods and patients The genotypes of polymorphic markers of genes-candidates (IL4, IL4R, IL13, IL33, IL6, TLR4, MMP9) were examined by Real-time PCR for a group of
131 children 5-17 y.o. (average age 10.9, 81 boys and 50 girls) with pollinosis and for a control group of 78 healthy children. $86 \%$ of children with pollinosis had any family history of allergy, $58 \%$ had one allergic parent $(33 \%$ - allergic mother, 25\% - allergic father); $20 \%$ had both allergic parents; $51 \%$ had some family history of pollinosis. $75 \%$ of patients had atopic dermatitis while being toddlers. The average age of pollinosis debut was $4.9 \pm 2.8$. The diagnosis of pollinosis was confirmed by the positive skin prick tests or by high level of specific IgE to tree or grass pollen in blood serum.

Results We reveal a statistically significant association of the rs1805010 of IL4R gene with pollinosis. The children with GG genotype of rs1805010 had increased risk of pollinosis compared with healthy controls $(\mathrm{P}=0.010 ; \mathrm{OR}=3.73$; $95 \%$ CI, 1.24 - 11.28). Moreover, there was a tendency to increase the allele $\mathrm{C}$ frequency of the rs1805015 of the IL4R gene in children with pollinosis $(\mathrm{OR}=1,81, \mathrm{p}=0,040)$. No significant association was found with other polymorphisms.

Conclusion The results of the present study revealed an association of cytokine gene polymorphism IL4R rs1805010 with pollinosis. Our results indicate a high predictive value of this polymorphic marker for the development of pollinosis in Russian pediatric population.

\section{GP6 POOR CONTROL OF ALLERGIC DISEASE AMONG ADOLESCENTS}

Miranda Crealey*, Aideen Byrne. Our Lady's Childrens Hospital Crumlin, Dublin, Ireland

\subsection{6/archdischild-2019-epa.73}

Background The prevalence of food allergy in adolescents varies with estimates of $2.3-4.5 \%$ in different populations $(1,2)$. This group are a vulnerable population. They are at greater risk of fatal anaphylaxis and fatal asthmatic episodes. In Ireland, they have a potential further risk in that most have not had an opportunity to see an allergist through childhood due to extremely limited access.

Aims To examine the presence of risk factors for poorly controlled allergic disease identifiable in adolescent patients on the routine waiting list (WL) in the allergy department.

Methods Adolescents attending their first allergy clinic appointment who were on the routine outpatient WL were included between September- December 2017. Clinical data pertaining to each adolescent's allergic disease was collected at this appointment. A scoring system was utilised to numerically grade the cause for concern for each adolescent after review in clinic. A $1 \mathrm{~A}$ point was awarded for each major concern identified and $\mathrm{a} 1 \mathrm{~B}$ point was awarded for each moderate concern identified.

Results Data was collected on 17 adolescents over the 3month period. The primary reason for referral in all patients was investigation of food allergy. Ten were referred by GPs, 5 by general paediatricians and 2 by dermatologists. 15/17 adolescents had between 1-3 criteria for major concern; uncontrolled asthma $(n=6)$, recurrent reactions while on the WL $(n=11)$, anaphylaxis while on WL $(n=3)$, no AAI prescribed $(n=4) .14 / 17$ had criteria for moderate concern; not carrying a prescribed in date AAI $(n=9)$, poorly controlled allergic rhinitis $(n=10)$, social isolation $(n=3)$. The highest score was $3 A$ $+1 \mathrm{~B}$ in 2 adolescents. 